\title{
Towards a cure for osteoporosis: the UK Royal Osteoporosis Society (ROS) Osteoporosis Research Roadmap
}

\author{
Nicholas C. Harvey ${ }^{1,2} \cdot$ Kenneth E. Poole ${ }^{3} \cdot$ Stuart H. Ralston ${ }^{4} \cdot$ Eugene V. McCloskey, $^{5,6}$. Caroline B. Sangan ${ }^{7}$. \\ Lauren Wiggins $^{7} \cdot$ Craig Jones $^{7} \cdot$ Neil Gittoes ${ }^{7,8} \cdot$ Juliet Compston $^{9} \cdot$ the ROS Osteoporosis and Bone Research \\ Academy Investigators
}

Received: 15 October 2021 / Accepted: 10 December 2021 / Published online: 6 January 2022

( ) International Osteoporosis Foundation and National Osteoporosis Foundation 2022

\section{Introduction}

Osteoporosis is characterised by decreased bone mass and disruption of bone microarchitecture, resulting in reduced bone strength and increased risk of fracture [1]. Approximately one in two women and one in five men will sustain one or more such fragility fractures (that is, occurring as a result of minimal trauma, for example a simple fall) in their remaining lifetime after the age of 50 years [2-4]. In the UK, approximately 549,000 new fragility fractures occur each year, including 105,000 hip fractures, 86,000 clinical vertebral fractures, and 358,000 fractures at other sites $[5,6]$. Fragility fractures often result in severe pain, disability, and

Nicholas C. Harvey

nch@mrc.soton.ac.uk

1 MRC Lifecourse Epidemiology Centre, University of Southampton, Southampton, UK

2 NIHR Southampton Biomedical Research Centre, University of Southampton and University Hospital, Southampton NHS Foundation Trust, Southampton, UK

3 Metabolic Bone Disease Unit, Addenbrooke's Hospital, Cambridge, UK

4 Centre for Genomic and Experimental Medicine, Institute of Genetics and Cancer, University of Edinburgh, Edinburgh, UK

5 Centre for Metabolic Bone Diseases, University of Sheffield, Sheffield, UK

6 MRC Versus Arthritis Centre for Integrated Research in Musculoskeletal Ageing, Mellanby Centre for Musculoskeletal Research, University of Sheffield, Sheffield, UK

7 Royal Osteoporosis Society, Bath, UK

8 Centre for Endocrinology, Diabetes and Metabolism (CEDAM), University of Birmingham, Birmingham, UK

9 Department of Medicine, Cambridge Biomedical Campus, University of Cambridge, Cambridge, UK reduction in quality of life; major fractures, particularly hip and vertebral fractures, are also associated with increased mortality [4]. Fragility fractures impose a huge economic burden on health and social services, with an estimated annual cost to the National Health Service (NHS) in excess of $£ 4.7$ billion [5, 6]; across Europe, the cost approaches $€ 55$ billion [6].

Despite major advances in the assessment of fracture risk and the development of effective pharmacological interventions to reduce fracture, many studies have documented a large treatment gap with only a minority of high-risk individuals receiving appropriate assessment and treatment [7-11]. Possible reasons for this include inadequate awareness and understanding of osteoporosis among the public and healthcare professionals, fear of adverse effects of drugs used in its treatment, and a lack of coordinated service delivery models for those affected [7]. Thus, it is clear that, in addition to addressing gaps in the understanding of the condition itself, removing barriers to appropriate assessment and treatment for all individuals at high risk of fragility fracture is a major priority.

The ROS Osteoporosis and Bone Research Academy was launched in February 2019 as part of the endowment of a royal title for the Charity, formerly known as the National Osteoporosis Society. The mission of the Academy, to work towards a cure for osteoporosis, is fully aligned with the Charity's vision of a future without fragility fractures and its four strategic aims of prevention, care, support, and cure. To this end, a Working Group structure was set up to address the themes of: causes and mechanisms; the use of novel technologies in diagnosis and management; and strategies to optimise the effectiveness of fracture risk assessment and treatment. The themes function synergistically and are supported by patient advocates, clinicians and scientists, with administrative and governance support from the ROS. Their outputs are overseen by an Advisory Committee, 
which reports to the ROS Board of Trustees. The high-level research strategy is set out in the Osteoporosis and Bone Research Academy's Osteoporosis Research Roadmap (https://view.publitas.com/royal-osteoporosis-society/rososteoporosis-research-roadmap-2021/page/1). In this editorial article, we summarise the approach taken to scoping and evaluating the existing literature, developing and planning a portfolio of scientific projects, and critical contribution from patient advocates. Detailed descriptions of individual areas have been published separately (functional genomics [12], opportunistic fracture detection [13]) or are in preparation (currently medication adherence, automated care pathways, osteoporosis screening, microbiome and bone health). The detailed exposition of the prior literature and planned projects is therefore beyond the scope of the overview presented in this editorial article.

\section{Methodology}

The initial phase of the Academy's programme has been focused on selecting research priorities and identifying the knowledge gaps in those topics. This has been achieved through expert-led consensus meetings and workshops, and the subsequent commissioning of focused rapid evidence reviews. The patient voice has been central to the development of the research priorities, principally through the work of the Academy Patient Advocates, and the prioritisation has been endorsed through a patient insight survey completed by 2313 public members of the charity, confirming alignment between the charity's strategic direction and the patient's lived experience. Specific projects selected as a result of this process are being undertaken in the second phase; funding to support this work is being sought from a variety of sources, all of which will be subject to rigorous peer review.

\section{Proposed work plans}

\section{Causes and mechanisms}

One of the most effective strategies for the prevention and treatment of a disease is to gain greater understanding of the underlying causes. While a great deal is known about predisposing factors for osteoporosis, there remain many areas where knowledge is incomplete, and these form the focus for the work of the Causes Working Group (CWG). Across the three subthemes of rare conditions, novel genetic and nongenetic causes, and new and better treatments, specific priorities include pregnancy-associated osteoporosis; genetic and epigenetic studies; interactions between diet, the microbiome, and bone health; and cellular senescence.
Pregnancy and lactation-associated osteoporosis (PAO) was identified as the CWG's foremost priority area for new research within the rare diseases subtheme. Understanding of PAO has been hindered by the rarity of the disorder, with research into the condition largely limited to small descriptive case series [14]. With the support of ROS infrastructure and pump priming funding, together with Academy Patient Advocates, the CWG established a UK-wide research study in which patients affected by the disorder can provide detailed information about risk factors, mode of presentation, and the treatment they received as well as its impact on quality of life. As part of this study, participants will have the opportunity to provide blood samples for genetic and biochemical analysis with the aim of establishing a PAOspecific Biobank. This initiative is expected to advance understanding of the disease and to inform the design of future randomised clinical trials to optimise bone health and prevent recurrence in subsequent pregnancies.

It has been long established that genetic factors play an important role in predisposition to osteoporosis but the predisposing variants have until recently been incompletely understood [12]. Advances in genotyping technology, coupled with the development of large-scale, intensively phenotyped cohort studies, have resulted in a step change in understanding the genetic basis of osteoporosis [15]. It is anticipated that this new knowledge can be harnessed to favourably impact on clinical outcomes in two ways: identification of molecular targets for drug design; and the development and implementation of polygenic risk scores (PRS) to stratify individuals at risk into routine clinical practice. An example of target identification already provided by GWAS is the finding that the glycosylation enzyme, B4GALNT3, is associated with both bone mineral density and serum sclerostin, thereby identifying this gene as a new target for drug design [16]. As exome and whole-genome sequencing data accrue across the entire UK Biobank cohort, the possibilities for detailed genetic characterisation through this work plan will expand considerably. Further research will need to be carried out to explore how best to use PRS-based approaches in clinical practice, but one potential avenue is to evaluate whether PRS has utility in the early identification of patients with severe osteoporosis of the spine, since these individuals often do not score highly on clinical risk factor calculators [17].

Research into the role of diet in bone health has traditionally focused on calcium and vitamin $\mathrm{D}$, but it has recently become apparent that the intestinal microbiome is also likely to play a key role [18]. Over the past five years, mechanistic studies in animal models $[19,20]$, observational studies in humans [21], and intervention studies have indicated that the microbiome significantly influences bone health [22]. Several gaps in knowledge remain that are amenable to future research, for example whether recent dietary trends 
(veganism, non-coeliac gluten-free diets) affect bone mineral density through microbiome-mediated pathways and whether diet-associated modifications of the gut microbiota impact bone loss.

The final area of emerging interest identified by the CWG as a determinant of bone health is cellular senescence. Preclinical research has shown that targeting senescence through genetic means or using drugs that inhibit the senescence-associated secretory phenotype (SASP) in preclinical models can improve physical function and extend lifespan, as well as inhibiting age-related bone loss [23, 24]. Furthermore, Janus-activated kinase (JAK) inhibitors which inhibit SASP have recently been shown to stimulate osteoblast function in preclinical models [25]. Phase I and early phase II clinical trials are currently in progress to examine the impact of pharmacological interventions aimed at inhibiting senescence on various phenotypes including bone health and thus may offer the potential for new and better treatments for osteoporosis.

\section{Novel technology for skeletal assessment}

Three key areas have been identified in the work plan for the technology theme. These priorities cover research to derive new measures of bone strength, for example from imaging or new assessment modalities; use of large datasets such as NHS patient records and UK Biobank to identify novel risk factors, for example delineating the contribution of muscle health to fracture risk and associated new targeted interventions; and, as the initial principal focus, the opportunistic detection of vertebral fractures on routine hospital imaging. While a vertebral fracture is an archetypal fracture of osteoporosis, it is also the least likely to come to clinical attention and receive formal assessment [4, 26, 27]. Patients often suffer from further multiple spine or other fractures, substantial deformity, height loss, and pain before diagnosis [7]. Technological approaches to finding vertebral fractures and taking appropriate action can be considered the first "mountain" that the Technology Working Group (TWG) have set out to climb, initially by compiling an evidence review to define the problem [13].

One way to improve both vertebral fracture identification and the diagnosis of osteoporosis is to assess a patient's spine or hips during routine computed tomography (CT) scans [28], an approach that is enthusing clinicians, software engineers, and patients alike. Patients attend routine CT for diagnosis and monitoring of various medical conditions, but the skeleton can be overlooked as radiologists concentrate on the primary reason for scanning. The more than six million CT scans undertaken each year in the NHS could potentially be screened for vertebral fractures and osteoporosis [13]. If CT-based case-finding became embedded in practice, then the technique could have a positive clinical impact. However, technology to find these sentinel vertebral fractures is only the "base camp" aim of the TWG mission.

To reach the summit requires Technology and Implementation research as we have laid out in our description of Jane's bone health journey, part of the group's 10-year vision. "In 2030, 72-year-old Jane attends hospital for a CT scan to investigate weight loss. She fills in a bone health (FRAX®) questionnaire at a touchscreen in the CT waiting room when checking in. An incidental osteoporotic vertebral fracture is identified automatically in the CT images by a computer-aided process, which also identifies that she has spinal osteoporosis in her lumbar vertebrae. The automatic inclusion of the smart phrase, 'There is an osteoporotic vertebral fracture' on the CT report spontaneously triggers four actions without human input: (1) Jane is sent a communication explaining the presence of an osteoporotic vertebral fracture and directing her to the Royal Osteoporosis Society website for more information on the diagnosis and selfmanagement. This communication is electronically copied to her GP; (2) She is automatically sent an appointment for a femoral neck bone density scan, and follow-up in the local Fracture Liaison Service, as per agreed local pathway; (3) She is automatically sent information on self-management to improve health-related quality of life (pain, posture, balance etc.) with a clear description of when to seek additional medical input (e.g. for onward referral for physio); and (4) An appointment is automatically made for Jane to see a healthcare professional (doctor, nurse, pharmacist) at her GP practice to discuss further, start on appropriate medication to reduce her risk of fracture, and be monitored to address any concerns and promote adherence. There is a clear potential risk of depersonalisation through such an automated process and thus throughout the process Jane will be able to obtain further information from a variety of sources with which to ensure confidence in the process."

This vision is entwined with the Effectiveness Working Group (EWG) mission and is also at the core of the Royal Osteoporosis Society Research Strategy, designed and endorsed by patients and researchers.

\section{Optimising effectiveness of assessment and treatment}

The importance of translating and implementing strategies and interventions that have well-proven efficacy into routine clinical practice is one of the biggest challenges in clinical medicine; the goal is to maximise the effectiveness of any high-value development in disease management [29]. Targeted interventions include, for instance, financial incentive programmes, continuing professional education, and, increasingly, tools to involve patients more actively in their care with shared decision-making. Despite educational initiatives, and relatively modest financial incentives in some healthcare settings, the management of 
osteoporosis and fracture risk remains sub-optimal. For example, the assessment and identification of those at high risk of fracture in primary care remain very low (approximately 25\%) [30], the long-term persistence and adherence with the mainstays of treatment, oral bisphosphonates, also remain poor [31], and finally there is a clear need to optimise the implementation of initiatives such as fracture liaison services (FLS) nationwide to further develop clinical systems and to ensure comprehensive dissemination and adoption of best practice [32].

These three areas have been identified as key work plan priorities of the EWG. At the primary care level, workshops have engendered conversations with patient representatives, primary care staff (GP and non-GPs), information technologists, primary care software providers, and regional health partnerships to share best practice and identify opportunities. Examples of the latter include the increasing use of health app technologies by patients with potential direct interaction with electronic patient records (EPRs); work is also planned to examine downstream pathways from the EPR back to the patient in terms of risk assessment, communication/information sharing and prescribing, where appropriate, with assessment of impact on primary care workloads. Problems with adherence are seen as part of a bigger issue, that of the need for improved follow-up processes and communication [33,34]. A rapid evidence review is being undertaken to summarise the current evidence to identify priority areas for research and implementation. Finally, in the area of FLS, there is increasing interest in the use of artificial intelligence techniques for automated image processing to detect vertebral fractures within routine clinical images (e.g. CT, MR, digital radiographs). This automated detection would then be integrated with FLS assessments and advice. Other initiatives will examine the ability of FLS to ensure rapid assessments and initiation of more appropriate therapies (e.g. bone-forming therapies) in those at very high risk.

There is, of course, much overlap in the areas identified by the other two Working Groups within the Academy; obvious examples include the enhanced use of technologies to streamline identification, initiation of treatment, and improved interaction with patients in the longer term, with particular emphasis on optimising medicines effectiveness, including promotion of adherence. The need for the EWG to work closely with experts in implementation science is also clear; our ultimate aim is for highly efficacious, proven interventions in osteoporosis to become more effective, thus leading to significantly improved care and outcomes for patients and the healthcare system.

\section{Discussion}

The proposed Academy work plans address three key facets of osteoporosis research and care. There is clear synergy across the Academy, for example in the use of big datasets such as UK Biobank and electronic patient records, and in the computational methods and novel technological approaches which are key to the majority of work plans across the three themes. Through close collaboration across Academy membership and involvement of adjunctive international expertise, the ROS Osteoporosis and Bone Research Academy presents the potential for step changes in osteoporosis research and care.

Previous publications have addressed the care gaps in osteoporosis service provision [7-11]. A position paper linked to the 2016 World Osteoporosis Day report from the International Osteoporosis Foundation described care gaps along four distinct themes: (1) case finding and management of individuals at high risk of fracture, (2) public awareness of osteoporosis and fragility fractures, (3) reimbursement and health system policy and (4) epidemiology of fracture in the developing world [7]. More recently, the International Osteoporosis Foundation released a further policy document, "Broken bones, broken lives" again documenting the burden of disease and deficits in service provision in the European Union [35]. These European initiatives are complemented by a secondary prevention focused "Call to Action" underpinned by an international consortium, including the UK Royal Osteoporosis Society, and led by the American Society for Bone and Mineral Research [11]. Whilst these initiatives highlight the gaps in osteoporosis care, they do not set out a consequent research agenda, rather focusing on healthcare and policy changes employing current evidence and technology.

To our knowledge, the ROS Osteoporosis Research Roadmap is the only such approach currently active, bringing together both identification of care gaps with research-focused solutions, and combining clinical and research expertise with the lived experience of the patient voice. The approach is broad, across clinical investigation, population health, fundamental mechanisms, and use of technology. It therefore differs from that set out by Yang et al., which is focused purely on molecular and genetic pathways [36]. A potential limitation of the ROS Academy methodology is the pragmatic, expert-led assessment of the broad existing evidence base. Whilst a systematic approach might have yielded a fully comprehensive review of the literature, initial scoping assessments suggested that it was highly unlikely that gaps with practicable solutions, unknown currently, would be identified. Thus, the approach undertaken was employed, and systematic rapid evidence reviews of individual areas used to ensure comprehensive coverage in those themes most likely to underpin achievable advances in the next 5-10 years.

In addition to the synergy between the research themes of the ROS Academy, a core component is the involvement of patient advocates in all aspects of the work. The patient voice is prominently represented through 
the cadre of Academy Patient Advocates, who between them sit on the Advisory Committee and on all the Working Groups. Thus, the work plans have been evaluated, advised upon, and approved by the Academy Patient Advocates; indeed the overall workplan was reviewed by the wider lay membership of the ROS, receiving strong endorsement of the research priorities. The onus is now on the UK osteoporosis research and clinical community, through the leadership of the ROS Academy, supported by global expertise, to draw in funding and support from a wide range of private, public, and corporate sources, in order to advance this mission. The research priorities identified by the Academy provide a rigorously evidenced thematic basis on which to build future ROS grant funding rounds, thus maximising the potential benefits for bone health.

In conclusion, the ROS Osteoporosis and Bone Research Academy, with its Osteoporosis Research Roadmap, presents a unique opportunity to achieve a step change in understanding of osteoporosis and clinical care provision. Optimisation of bone health across the lifecourse, at the population level, and ensuring that every individual at high risk of fragility fracture is appropriately identified, assessed, and treated, will underpin substantial progression towards the ROS mission of achieving a world without fragility fractures.

Abbreviations CT: Computed tomography; CWG: Causes Working Group; EPR: Electronic patient record; EWG: Effectiveness Working Group; FLS: Fracture liaison service; GP: General practitioner; GWAS: Genome-wide association study; JAK: Janus-activated kinase; NHS: National Health Service; PAO: Pregnancy and lactation-associated osteoporosis; PRS: Polygenic risk scores; ROS: Royal Osteoporosis Society; SASP: Senescence-associated secretory phenotype; TWG : Technology Working Group

Acknowledgements We are hugely grateful to the Royal Osteoporosis Society staff for their continued support of the ROS Academy, in particular Lauren Wiggins, Caroline Sangan, Jamie Grier, Craig Jones, and previously Belinda Thompson, Francesca Thompson, Fizz Thompson, and Claire Severgnini, together with the invaluable contribution of the ROS Academy Patient Advocates, and in particular Nic Vine and Mary Bishop for the detailed review and feedback on this article.

\section{ROS Osteoporosis and Bone Research Academy Investigators:}

\section{Advisory Committee}

Prof Juliet Compston (Chair), Prof Nicholas C Harvey (Vice Chair), Prof Cyrus Cooper, Prof Neil Gittoes, Prof Eugene McCloskey, Dr Nicky Peel, Dr Kenneth Poole, Prof Stuart H Ralston, Prof David Reid, Prof Graham Russell (past member), Prof Jon Tobias, Prof Bo Abrahamsen, Prof Richard Eastell; Patient Advocates: Mrs Mary Bishop and Mr Nic Vine

Causes Working Group

Prof Stuart H Ralston (Chair), Dr Rosemary Hollick (Vice Chair), Dr Owen Cronin, Prof Emma Duncan, Prof Celia Gregson, Dr Nuria Gunabens, Prof Fadil Hannan (past member), Dr Richard Keen, Prof Susan
Lanham-New, Prof Tim Spector, Prof Jon Tobias; Patient Advocates: Mrs Joy Capel and Mrs Karen Whitehead

Effectiveness Working Group

Prof Eugene McCloskey (Chair), Dr Zoe Paskins (Vice Chair), Dr David Armstrong, Dr Elizabeth Curtis, Dr John Edwards, Dr Stephen Gallacher (past member), Prof Thomas Hill, Dr Kassim Javaid, Prof Terry O’Neill, Prof Michael Stone, Dr Jennifer Walsh; Patient Advocates: Ms Janice McKinley and Ms Corinne Turnbull

Technology Working Group

Dr Kenneth Poole (Chair), Prof Emma Clark (Vice Chair), Dr Richard Abel, Dr Pinaki Bhattacharya, Dr Paul Bromiley, Dr Nicola Crabtree, Dr Jennifer Gregory, Prof Nicholas C Harvey, Dr Eleni Kariki, Prof Kate Ward; Patient Advocates: Miss Lois Ainger and Mrs Karen Whitehead

\section{Declarations}

Ethics statement This narrative article contains no original data and thus issues of ethics, informed consent, and patient confidentiality do not apply.

Conflicts of interest NCH reports personal fees, consultancy, lecture fees, and honoraria from Alliance for Better Bone Health, AMGEN, MSD, Eli Lilly, Servier, Shire, UCB, Consilient Healthcare, Kyowa Kirin, and Internis Pharma, outside the submitted work. JC has received lecture fees from Amgen and UCB and consultancy fees from Amgen. EVM reports personal fees, consultancy, lecture fees, and/or honoraria from Amgen, Consilient Healthcare, Fresenius Kabi, GSK, Hologic, Internis, ObsEva, Roche, Sanofi-Aventis, Servier, and UCB, all outside the submitted work. All other authors declared no conflicts of interest.

\section{References}

1. Anon (1993) Consensus development conference: diagnosis, prophylaxis, and treatment of osteoporosis. Am J Med 94:646-650

2. van Staa TP, Dennison EM, Leufkens HG, Cooper C (2001) Epidemiology of fractures in England and Wales. Bone 29:517-522

3. Curtis EM, van der Velde R, Moon RJ, van den Bergh JP, Geusens P, de Vries F, van Staa TP, Cooper C, Harvey NC (2016) Epidemiology of fractures in the United Kingdom 1988-2012: variation with age, sex, geography, ethnicity and socioeconomic status. Bone 87:19-26

4. Harvey N, Dennison E, Cooper C (2010) Osteoporosis: impact on health and economics. Nat Rev Rheumatol 6:99-105

5. Borgström F, Karlsson L, Ortsäter G et al (2020) Fragility fractures in Europe: burden, management and opportunities. Arch Osteoporos 15:59

6. Kanis JA, Norton N, Harvey NC, Jacobson T, Johansson H, Lorentzon M, McCloskey EV, Willers C, Borgström F (2021) SCOPE 2021: a new scorecard for osteoporosis in Europe. Arch Osteoporos $16: 82$

7. Harvey NC, McCloskey EV, Mitchell PJ, Dawson-Hughes B, Pierroz DD, Reginster JY, Rizzoli R, Cooper C, Kanis JA (2017) Mind the (treatment) gap: a global perspective on current and future strategies for prevention of fragility fractures. Osteoporos Int 28:1507-1529

8. Khosla S, Cauley JA, Compston J, Kiel DP, Rosen C, Saag KG, Shane E (2017) Addressing the crisis in the treatment of osteoporosis: a path forward. J Bone Miner Res 32:424-430

9. Compston J (2020) Reducing the treatment gap in osteoporosis. Lancet Diabetes Endocrinol 8:7-9 
10. Harvey NCW, McCloskey EV, Rizzoli R, Kanis JA, Cooper C, Reginster J-Y (2019) Osteoporosis: treatment gaps and health economics. In: Huhtaniemi I, Martini L (eds) Encyclopedia of endocrine diseases, 2nd edn. Academic Press, Oxford, pp 288-295

11. Conley RB, Adib G, Adler RA et al (2020) Secondary fracture prevention: consensus clinical recommendations from a multistakeholder coalition. J Orthop Trauma 34:e125-e141

12. Tobias JH, Duncan EL, Kague E et al (2020) Opportunities and challenges in functional genomics research in osteoporosis: report from a workshop held by the Causes Working Group of the Osteoporosis and Bone Research Academy of the Royal Osteoporosis Society on October 5th 2020. Front Endocrinol 11:630875

13. Aggarwal V, Maslen C, Abel RL et al (2021) Opportunistic diagnosis of osteoporosis, fragile bone strength and vertebral fractures from routine CT scans; a review of approved technology systems and pathways to implementation. Ther Adv Musculoskelet Dis $13: 1759720 \times 211024029$

14. Hardcastle SA (2021) Pregnancy and lactation associated osteoporosis. Calcif Tissue Int; Online ahead of print.

15. Morris JA, Kemp JP, Youlten SE et al (2019) An atlas of genetic influences on osteoporosis in humans and mice. Nat Genet $51: 258-266$

16. Zheng J, Maerz W, Gergei I et al (2019) Mendelian randomization analysis reveals a causal influence of circulating sclerostin levels on bone mineral density and fractures. J Bone Miner Res 34:1824-1836

17. Oswald AJ, Berg K, Ralston SH, Riches PL (2019) Long-term effects of teriparatide followed by antiresorptive therapy on clinical outcomes in patients with severe spinal osteoporosis. Calcif Tissue Int 105:148-155

18. Ohlsson C, Sjogren K (2018) Osteomicrobiology: a new crossdisciplinary research field. Calcif Tissue Int 102:426-432

19. Sjogren K, Engdahl C, Henning P, Lerner UH, Tremaroli V, Lagerquist MK, Backhed F, Ohlsson C (2012) The gut microbiota regulates bone mass in mice. J Bone Miner Res 27:1357-1367

20. Li JY, Chassaing B, Tyagi AM et al (2016) Sex steroid deficiencyassociated bone loss is microbiota dependent and prevented by probiotics. J Clin Invest 126(6):2049-2063

21. Das M, Cronin O, Keohane DM et al (2019) Gut microbiota alterations associated with reduced bone mineral density in older adults. Rheumatology (Oxford) 58(12):2295-2304

22. Nilsson AG, Sundh D, Backhed F, Lorentzon M (2018) Lactobacillus reuteri reduces bone loss in older women with low bone mineral density: a randomized, placebo-controlled, double-blind, clinical trial. J Intern Med 284:307-317

23. Xu M, Pirtskhalava T, Farr JN et al (2018) Senolytics improve physical function and increase lifespan in old age. Nat Med $24: 1246-1256$
24. Farr JN, Xu M, Weivoda MM et al (2017) Targeting cellular senescence prevents age-related bone loss in mice. Nat Med 23:1072-1079

25. Adam S, Simon N, Steffen U et al (2020) JAK inhibition increases bone mass in steady-state conditions and ameliorates pathological bone loss by stimulating osteoblast function. Sci Transl Med 12(530):eaay 4447

26. Compston J, Cooper A, Cooper C et al (2017) UK clinical guideline for the prevention and treatment of osteoporosis. Arch Osteoporos $12: 43$

27. Kanis JA, Cooper C, Rizzoli R, Reginster JY (2019) European guidance for the diagnosis and management of osteoporosis in postmenopausal women. Osteoporos Int 30:3-44

28. Bromiley PA, Clark EM, Poole KE (2020) Computer-aided diagnostic systems for osteoporotic vertebral fracture detection: opportunities and challenges. J Bone Miner Res 35:2305-2306

29. Wensing M, Grol R (2019) Knowledge translation in health: how implementation science could contribute more. BMC Med 17:88

30. McCloskey E, Rathi J, Heijmans S et al (2021) The osteoporosis treatment gap in patients at risk of fracture in European primary care: a multi-country cross-sectional observational study. Osteoporos Int 32:251-259

31. Fatoye F, Smith P, Gebrye T, Yeowell G (2019) Real-world persistence and adherence with oral bisphosphonates for osteoporosis: a systematic review. BMJ Open 9:e027049

32. Javaid MK (2021) Efficacy and efficiency of fracture liaison services to reduce the risk of recurrent osteoporotic fractures. Aging Clin Exp Res 33:2061-2067

33. Hawarden A, Jinks C, Mahmood W, Bullock L, Blackburn S, Gwilym S, Paskins Z (2020) Public priorities for osteoporosis and fracture research: results from a focus group study. Arch Osteoporos 15:89

34. Paskins Z, Jinks C, Mahmood W, Jayakumar P, Sangan CB, Belcher J, Gwilym S (2017) Public priorities for osteoporosis and fracture research: results from a general population survey. Arch Osteoporos 12:45

35. (2018) BROKEN BONES, BROKEN LIVES: A roadmap to solve the fragility fracture crisis in Europe. International Osteoporosis Foundation, Nyon

36. Yang TL, Shen H, Liu A, Dong SS, Zhang L, Deng FY, Zhao Q, Deng HW (2020) A road map for understanding molecular and genetic determinants of osteoporosis. Nat Rev Endocrinol 16:91-103

Publisher's note Springer Nature remains neutral with regard to jurisdictional claims in published maps and institutional affiliations. 